\title{
Semantic similarity and immediate serial recall: Is there an effect on all trials?
}

\author{
JEAN SAINT-AUBIN and DENIS OUELLETTE \\ Université de Moncton, Moncton, New Brunswick, Canada \\ and \\ MARIE POIRIER \\ City University, London, England
}

\begin{abstract}
In immediate serial recall, items are better recalled when they are all drawn from the same semantic category. This is usually accounted for by a two-stage retrieval-based framework, in which, at recall, long-term knowledge is used to reconstruct degraded phonological traces. The category shared by list items would serve as an additional retrieval cue restricting the number of recall candidates. Usually, the long-term search set is not defined, but some authors have suggested an extended search set and others a restricted set that is composed of the most recently presented items. This was tested in an experiment in which participants undertook an immediate serial recall task either alone or under articulatory suppression with either semantically similar or dissimilar lists. A trial-by-trial analysis revealed that, in both quiet and suppression conditions, items from similar lists were better recalled on all the trials, including the first one. In addition, there was no interaction between semantic similarity and trial, indicating that the effect of similarity was of similar size on all the trials. The results are best interpreted within a proposal suggesting an extended long-term search set.
\end{abstract}

In immediate serial recall, it is well established that long-term memory factors, such as lexicality, word frequency, and semantic similarity, contribute to performance (see, e.g., Hulme, Maughan, \& Brown, 1991; Multhaup, Balota, \& Cowan, 1996; Murdock, 1976; Poirier \& SaintAubin, 1995; Stuart \& Hulme, 2000). To account for these effects, a reconstruction hypothesis is often put forward (see, e.g., Hulme et al., 1991; Saint-Aubin \& Poirier, 1999, 2000; Schweickert, 1993). This hypothesis can account for the effects of most, if not all, long-term memory factors recently investigated in this field by suggesting that long-term knowledge of the to-be-remembered items is called upon at retrieval. However, as of yet, in the context of the reconstruction hypothesis, the nature of the set of items called upon at recall has not been clearly defined. The aim of the present study was to investigate this question by taking advantage of the semantic similarity effect. Because of the trial-by-trial analysis that we pursued, the data set is also of interest in terms of constraining models accounting for performance in shortterm memory tasks.

The reconstruction hypothesis can be described as follows. At recall, phonological representations set up by

This research was supported by a discovery grant from the Natural Sciences and Engineering Research Council of Canada to J.S.-A. We thank Ian Neath, Gerry Tehan, and Sébastien Tremblay for their helpful comments on earlier versions of this article. Correspondence concerning this article should be addressed to J. Saint-Aubin, Département de Psychologie, Université de Moncton, Moncton, NB, E1A 3E9 Canada (e-mail: saint-aj@umoncton.ca). list presentation are thought to be degraded and undergo a reconstruction process calling upon long-term knowledge of the to-be-remembered items. Broadly put, longterm knowledge of the language would be called upon to fill in the gaps left by degradation. Typical short-term memory factors, such as word length and articulatory suppression, are thought to influence the amount of degradation (Neath \& Nairne, 1995; Saint-Aubin \& Poirier, 2000). On the other hand, long-term memory factors are thought to influence the accessibility of the recall candidates (Saint-Aubin \& Poirier, 1999, 2000; Schweickert, 1993; Stuart \& Hulme, 2000).

Although the reconstruction hypothesis has been successful in accounting for the influence of long-term memory factors on immediate serial recall performance, in most versions of the hypothesis the memory search set called upon at retrieval has not been clearly defined. In fact, Brown and Hulme (1995) argued that within the reconstruction hypothesis there is an "absence of any specification of the "vocabulary' [quotation marks in the original] used during the redintegration process. Are partially decayed traces compared to every possible vocabulary item? To every item that has been used in the experimental situation?" (p. 600). Multhaup et al. (1996) suggested that the reconstruction hypothesis "does assume that when subjects perform a word span task, they restrict their search of long-term memory information to the items on the to-be-remembered list" (p. 114). Similarly, in the first version of the feature model, Nairne (1990) suggested that the secondary memory vector used for reconstruction was limited to the presented items within a list. 
This suggestion seems unlikely for a number of reasons. For example, although this is relatively infrequent, participants do sometimes recall a word presented on a previous trial or from outside the experimental situation (see, e.g., Saint-Aubin \& Poirier, in press; Stuart \& Hulme, 2000). Furthermore, intrusions from previous trials appear to obey a number of rules and, as such, could not be due to a simple guessing strategy (see, e.g., Estes, 1991; Tehan \& Humphreys, 1995; Tolan \& Tehan, 1999).

An alternative is to argue that the memory search set is composed of recently presented items. For instance, Hulme, Stuart, Brown, and Morin (2003) "assume that following list presentation a strictly limited set of items (consisting of the items presented on that trial, plus perhaps some of the items presented on other recent trials) is available in a retrievable state" (p. 514). Neath (1994; Neath \& Nairne, 1995) goes even further by suggesting a modification to the feature model. According to this modification, the search is not restricted to the items presented on the current trial-as in the original version of the feature model - or to the items presented on recent trials. More precisely, it is suggested that participants make inferences about plausible recall candidates. If, on one trial, items are all vegetable names, all kinds of vegetables are potential recall candidates. Otherwise, the search set could be larger. Neath reported simulations in which the size of the memory search set was manipulated to reflect the participant's ability to make inferences about plausible candidates. Recall performance varied as a function of memory search set size.

Semantic similarity is a well-suited factor for investigating the memory search set called upon at recall. It is usually assumed that the semantic category shared by list items in a semantically similar list could be used as an additional retrieval cue, which restricts the number of recall candidates (see, e.g., Crowder, 1979; Neath, 1994; Poirier \& Saint-Aubin, 1995; Saint-Aubin \& Poirier, 1999). In support of this view, Roodenrys and Quinlan (2000) showed that repeating the same items on every trial (in a different order) produced better performance than did using different items on every trial. They suggested that the repeated presentation of the words from the closed set condition resulted in the priming of their representations in long-term memory, giving these words an advantage during redintegration. In effect, it can be argued that the repeated presentation of a small set of words within a specific experimental context creates an ad hoc category that, in the words of Roodenrys and Quinlan, "reduces the number of words that will play a part in the competition during the reconstructive process" (p. 77; see also Saint-Aubin \& Poirier, in press). It is worth mentioning that we are not suggesting that semantic similarity is the only factor that could restrict the search set. For instance, the search set could also be restricted by other factors, such as temporal information, perhaps along the lines suggested by the temporal distinctiveness hypothesis (e.g., Neath \& Crowder, 1990).

If, as has been assumed by Hulme et al. (2003), the memory search set is composed of recently presented items, a certain number of predictions can be made. More specifically, for lists of items from different categories, on the first trial, the search set would be smaller than on subsequent trials. Consequently, we would expect performance to decline over trials for the dissimilar condition. However, for semantically similar lists, performance should not be as adversely affected. This would happen because items from recent trials would automatically be discarded from the search set on the basis of category membership. Consequently, this conception of the memory search set predicts an interaction between semantic similarity and trial order: Semantic similarity effects should be smaller on the first trial than on subsequent trials.

With regard to the similarity effect on the first trial, it can be hypothesized that semantically similar and dissimilar lists should be recalled to the same level. This would be the case if it is assumed that semantic similarity provides an additional retrieval cue that restricts the memory search set. Because the memory search set would already be limited to items presented on the current trial, the additional retrieval cue provided by category membership would be superfluous.

On the other hand, if it is assumed that the memory search set is not restricted to the most recently presented items - as is the case in the latest version of the feature model - then semantically similar lists should be better recalled than dissimilar lists on all the trials, including the first (Neath, 1994; Neath \& Nairne, 1995). In addition, performance should remain roughly the same across trials for both similar and dissimilar lists.

Finally, it could be argued that set size effects could be masked by the high quality of phonological representations in a standard immediate serial recall task. More precisely, in a series of studies in which various short-term ordered recall tasks were used, Tehan and Humphreys (1995; Tolan \& Tehan, 1999) demonstrated proactive interference effects when phonological codes were not present, degraded, or nondiscriminatory. In the work reported here, an articulatory suppression condition was included in order to test the hypothesis that the influence of the set size or of proactive interference would be restricted to situations in which phonological representations are less useful. If proactive interference occurs only when phonological representations are degraded, performance should be roughly constant across trials for both similar and dissimilar lists under a quiet condition. However, under suppression, because phonological representations are degraded, there should be proactive interference for dissimilar lists. Similar lists should be immune from proactive interference because the semantic category shared by all the list items changes from trial to trial.

\section{METHOD}

\section{Participants}

A total of 252 unpaid undergraduate students from the Université de Moncton (183 women and $69 \mathrm{men}$ ) volunteered to participate in this experiment. All were native French speakers. 


\section{Materials}

All the stimuli were presented in French. Fourteen semantically similar lists were assembled. Each list comprised 7 words drawn from the same semantic category (e.g., sports, vegetables, or trees), with most words having two syllables. From this pool of 98 words (14 lists of 7 words each), 14 dissimilar lists were assembled by sampling words without replacement. Therefore, each word was used once in a semantically similar list and once in a semantically dissimilar list. The following constraints were applied to the construction of the dissimilar lists. First, each word in a dissimilar list had to come from a distinct semantic category. Second, each dissimilar list was yoked to a similar list, so that both lists were equivalent in terms of word length and frequency. Third, dissimilar lists were assembled in such a way that each of the 7 words from a similar list was assigned to a different serial position in the dissimilar lists. For example, across the 7 dissimilar lists in which there was a vegetable name, there was a vegetable name in the first serial position in one dissimilar list, in the second serial position in another list, in the third in another one, and so on. In addition, in both similar and dissimilar lists, care was taken to avoid phonological similarity by not including words that rhymed or shared the first syllable.

\section{Design and Procedure}

There were two between-subjects factors: semantic similarity (similar vs. dissimilar) and viewing condition (quiet vs. suppression). There were 70 participants in each semantic similarity condition under the quiet condition and 56 per similarity condition under suppression. Within each of the four conditions, presentation order of the lists was counterbalanced with a Latin-square design. Consequently, across participants, a given list was presented equally often at each of the 14 possible trial positions. There was no practice trial.

The participants were tested individually within one session lasting about $30 \mathrm{~min}$. They sat in front of a computer, about $50 \mathrm{~cm}$ from the screen. The participants self-initiated a trial by pressing the space bar. Each trial began with the warning prompt "Attention!" (1,500 msec on, $500 \mathrm{msec}$ off). Seven words were then presented sequentially, in standard lowercase lettering (1,500 msec on, $500 \mathrm{msec}$ off $)$. After the last word, a row of asterisks was displayed as a recall prompt, remaining on the screen until the participant initiated the next trial.

Strict serial recall instructions were used. The participants were told to write the items in their exact order of presentation, beginning with the first one. They were instructed to leave a blank line if they could not recall an item at a given serial position. They were also warned not to backtrack to fill a blank. There was no time limit for recall. The experimenter was present throughout to ensure compliance with these instructions.

In the articulatory suppression condition, the participants continuously repeated aloud the word mathématiques, at a rhythm of about three utterances every $2 \mathrm{sec}$. The participants began suppressing as soon as they initiated a trial, and they continued until recall was completed.

\section{RESULTS AND DISCUSSION}

In order to allow comparison with previous studies, the first set of analyses was based on data from all 14 trials in each condition. These analyses included strict serial scoring, as well as order error analyses. The second set of analyses presented is a trial-by-trial analysis of item information recall. In all analyses, the .05 level of significance was adopted.

\section{Global Analyses}

Responses were first scored with a strict serial recall criterion. Accordingly, words had to be recalled in their exact presentation order to be considered correct. The probability of correct recall as a function of serial position, suppression, and similarity condition is shown in Figure 1 . This figure reveals a similarity advantage and a suppression impairment. In addition, the effects of semantic similarity and suppression seem larger on the first positions. A $2 \times 2 \times 7$ mixed design analysis of variance (ANOVA) with semantic similarity and suppression as between-subjects factors and serial position as the repeated factor confirmed those trends, with a significant main effect of semantic similarity $[F(1,248)=$ $\left.41.88, M S_{\mathrm{e}}=0.0668\right]$, of suppression $[F(1,248)=292.28$, $\left.M S_{\mathrm{e}}=0.0668\right]$, and of serial position $[F(6,1488)=$ 753.76, $\left.M S_{\mathrm{e}}=0.0193\right]$. In addition, there were two significant interactions. The first one was between similarity and serial position $\left[F(6,1488)=7.69, M S_{\mathrm{e}}=0.0193\right]$. Simple main effect tests revealed that the effect of similarity was significant on the first four serial positions, but not on the last three. There was also a significant interaction between suppression and serial position $[F(6,1488)=$ 47.05, $\left.M S_{\mathrm{e}}=0.0193\right]$. Simple main effect tests revealed

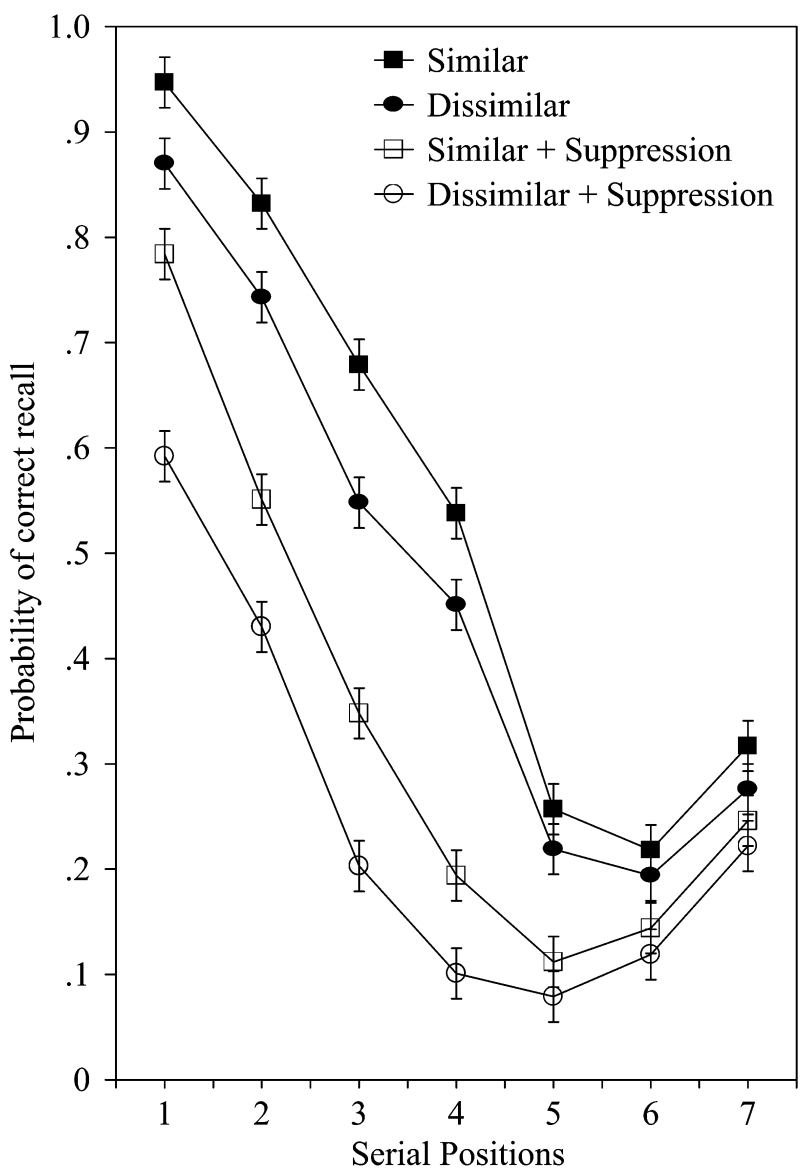

Figure 1. Mean probabilities of correct recall with a strict serial recall criterion as a function of semantic similarity, articulatory suppression, and serial position; error bars represent confidence intervals at $\alpha=.05$ for the between-subjects factors (similarity and suppression), computed according to the method of Loftus and Masson (1994). 
that the effect of suppression was significant on all serial positions but the last one.

The results with strict serial scoring are very similar to those in previous studies in which there was a similarity advantage under both quiet and suppression conditions, as well as, in some cases, an interaction with serial positionswith an advantage for similar lists restricted to or larger on the first serial positions (Brooks \& Watkins, 1990; Crowder, 1979; Poirier \& Saint-Aubin, 1995; Saint-Aubin \& Poirier, 1999). Because strict serial scoring factors in item and order information and because semantic similarity produces distinct effects on those two recall components, error analyses are more important for shedding light on the memory processes highlighted by semantic similarity.

Proportions of order errors were then computed by dividing the number of presented items that were not recalled at the appropriate serial position by the total number of items correctly recalled, regardless of their recall position accuracy. As is shown in Figure 2, proportions of order errors are higher for similar than for dissimilar lists and for the suppression than for the quiet condition. A $2 \times 2$ between-subjects ANOVA with similarity and suppression as factors confirmed these trends, with significant main effects of semantic similarity $\left[F(1,248)=30.10, M S_{\mathrm{e}}=\right.$ $0.0117]$ and of suppression $\left[F(1,248)=9.10, M S_{\mathrm{e}}=\right.$ $0.0117]$. The interaction did not reach significance.

The higher proportion of order errors under suppression is the standard finding in the field, and it could be accounted for by most models involving an account for short-term recall (see, e.g., Neath \& Nairne, 1995; SaintAubin \& Poirier, 2000). On the other hand, the higher proportion of order errors for semantically similar lists is surprising. In effect, we previously argued that when properly measured - as is the case here with the usage of proportion of order errors - the proportion of order errors should be equivalent for semantically similar and dissimilar lists (Saint-Aubin \& Poirier, 1999). A careful examination of previous studies in which order errors were properly measured and no similarity decrement was found revealed that at the descriptive level, there was almost always a trend toward a higher proportion of order errors for semantically similar items (Murdock, 1976; Saint-Aubin \& Poirier, 1999). Because of the very large number of participants in the present study, the small nonsignificant trend in previous studies was significant here. The theoretical implications of this finding will be discussed along with results of the trial-by-trial analysis.

\section{Trial-by-Trial Analyses}

For each condition and each trial, item information recall was analyzed as a function of trial order by computing the number of items correctly recalled with a free recall criterion, which was obtained by subtracting the total number of item errors from the number of presented items in a list - namely, 7. As is shown in Figure 3, on all trials, including the first one, there was an advantage of similar over dissimilar lists under both quiet and suppression conditions. Similarly, on all trials, there was a detrimental effect of articulatory suppression. Performance was similar over the 14 trials, and there were no systematic variations in the size of either the similarity or the suppression effect over trials. The $2 \times 2 \times 14$ mixed design ANOVA with similarity and suppression as between-subjects factors and trial as the repeated factor confirmed those trends. There was a significant effect of similarity $\left[F(1,248)=175.19, M S_{\mathrm{e}}=6.2491\right]$ of suppression $\left[F(1,248)=405.22, M S_{\mathrm{e}}=6.2491\right]$, and of trial $\left[F(13,3224)=1.86, M S_{\mathrm{e}}=1.0224\right]$. Post hoc comparisons (Tukey's HSD) revealed that the first trial was better recalled than the third and the fourth. No other pair of trials significantly differed. Because variations in performance level across trials were minimal, did not show a coherent trend, and did not interact with similarity, this effect is not especially relevant from a theoretical viewpoint and will not be further discussed. There was only one significant interaction, between trial and suppression $\left[F(13,3224)=1.96, M S_{\mathrm{e}}=1.0224\right]$. Simple main effect tests indicated that the effect of suppression was significant on all the trials. In addition, although the interaction

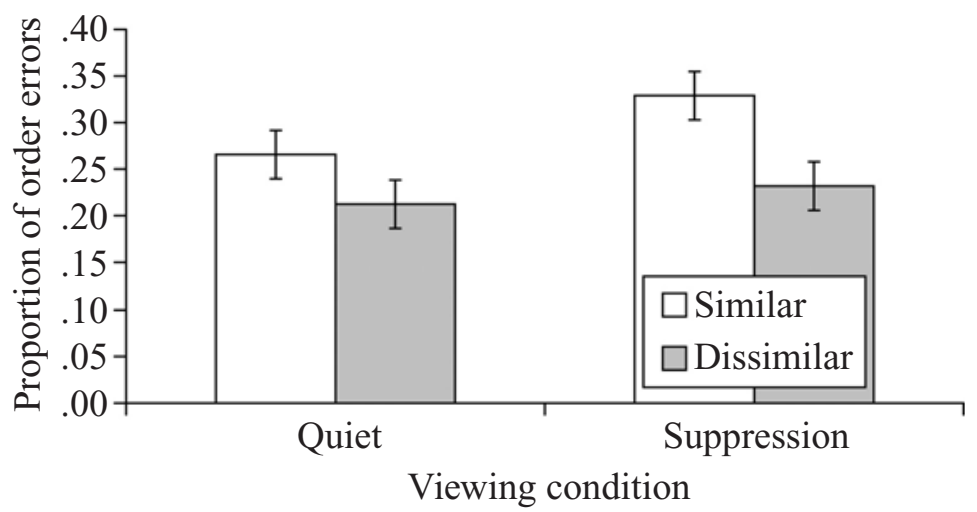

Figure 2. Mean proportions of order errors as a function of semantic similarity and articulatory suppression; error bars represent confidence intervals at $\alpha=.05$. 


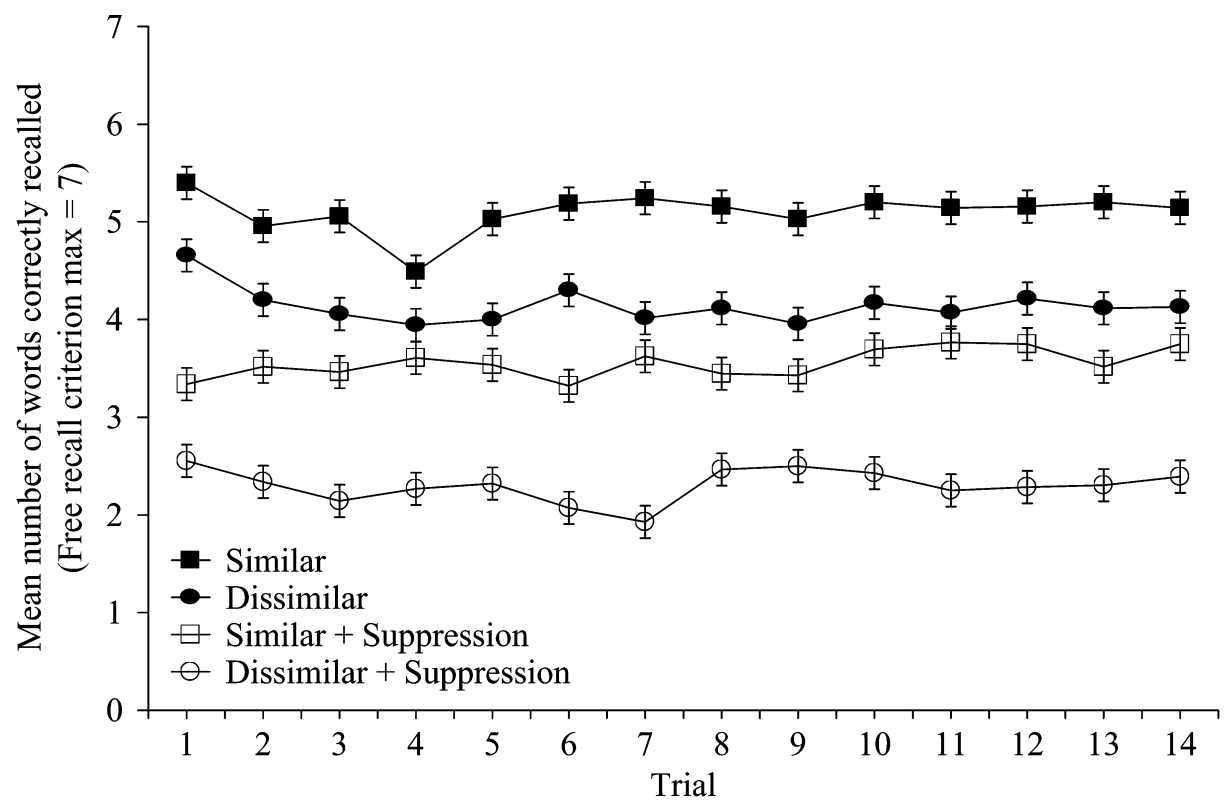

Figure 3. Mean probability of correct recall with a free recall criterion as a function of semantic similarity, articulatory suppression, and trial; error bars represent confidence intervals at $\alpha=.05$ for the between-subjects factors (similarity and suppression).

between similarity and trial did not reach significance, given its theoretical importance, planned contrasts were computed. Results revealed a significant advantage of similar lists on all the trials, including the first one.

Results of the trial-by-trial analysis are critical for evaluating hypotheses about the memory search set. The superior recall performance of similar over dissimilar lists on the first trial is difficult to account for by assuming that the memory search set is composed of recently presented items and that semantic similarity has a beneficial effect only by restricting the memory search set. In addition, the similar recall performance across trials for both similar and dissimilar lists and the fact that the similarity effect is constant across trials are difficult to account for by assuming that the memory search set is composed of recently presented items or that proactive interference is exerting a significant influence in immediate serial recall (e.g., Hulme et al., 2003).

The lack of evidence for proactive interference effects among dissimilar lists under both quiet and suppression conditions calls for comment. Under quiet conditions, as was mentioned in the introduction, participants do sometimes recall an item from previous trials. What is more, these intrusions from previous trials obey certain rules. For instance, when an item from trial $n-1$ is recalled on trial $n$, it is more likely to be recalled at the recall position of trial $n-1$ than at other positions (Estes, 1991). This could be taken as evidence for proactive interference. How could errors like these be reconciled with the present results showing no performance decrement across trials for dissimilar lists? Two points can be called upon to explain this apparent contradiction. First, intrusions from previous trials are very infrequent in immediate serial recall. For instance, in the standard conditions in Saint-Aubin and Poirier (in press), on average, there was one intrusion from a previous trial every seven lists (see Stuart \& Hulme, 2000, for similar results). The latter infrequent intrusions are a form of proactive interference and provide invaluable information for testing theories. However, contrary to what has been shown in other paradigms, in immediate serial recall, proactive interference does not seem to exert a large influence on recall performance.

As has been suggested by Tehan and Humphreys (1995; Tolan \& Tehan, 1999), the small magnitude of proactive interference effects in immediate serial recall could be due to the high quality of phonological representations. In this context, proactive interference effects should be much larger under suppression, because phonological representations are degraded. However, even under suppression, there was no indication of proactive interference for dissimilar lists, because recall performance was similar across trials. The discrepancy between our results and those in Tehan and Humphreys is likely to be due to methodological differences. ${ }^{1}$ In short, it seems that proactive interference effects in short-term ordered recall might be limited to situations in which at least two competitors are activated by the retrieval cue, which is not usually the case in immediate serial recall but is the case in Tehan and Humphreys's work.

On the other hand, the present results are compatible both with a version of the reconstruction hypothesis suggesting that a large memory search set is called upon at recall (Hulme et al., 1991; Stuart \& Hulme, 2000) and as with the feature model (Neath, 1994; Neath \& Nairne, 
1995). According to the reconstruction hypothesis, similar lists would be better recalled than dissimilar lists because, at recall, the category shared by list items would increase the probability of retrieving the appropriate long-term representations, either because the category would provide an additional retrieval cue (e.g., Crowder, 1979; Poirier \& Saint-Aubin, 1995; Saint-Aubin \& Poirier, 1999) or because long-term representations of similar items would be more activated due to their long-term associative links (Hulme et al., 2003; Stuart \& Hulme, 2000 ). This process would predict better item recall for semantically similar lists on all trials, including the first one. The lower recall under suppression would be attributable to the greater degradation of phonological traces. Because phonological traces are viewed as the basic retrieval cues, their greater degradation would translate into lower item recall (Saint-Aubin \& Poirier, 1999, 2000). According to the reconstruction hypothesis, there should also have been an interaction between semantic similarity and articulatory suppression. In short, because phonological representations are more degraded under suppression, the reconstruction process would have more impact (see Schweickert, 1993, for a formal demonstration). Descriptive results are in line with those predictions, with, on average, an advantage of 1.25 words recalled per list for semantically similar over dissimilar lists under suppression, which advantage was only 0.99 in the quiet condition. However, contrary to previous studies, the interaction here did not reach significance (Poirier \& Saint-Aubin, 1995; Saint-Aubin \& Poirier, 1999).

Finally, the higher proportion of order errors for similar lists is not compatible with previous versions of the reconstruction hypothesis, assuming that the degraded representations used as retrieval cues contain only phonological representations (e.g., Saint-Aubin \& Poirier, 1999). However, if it is assumed that the degraded phonological representations used as retrieval cues also contain a number of semantic features, it would be possible to account for the small detrimental effect of semantic similarity on order recall (see Neath \& Nairne, 1995, and Tolan \& Tehan, 1999, for similar ideas). In this case, common semantic features would hinder order recall by increasing the similarity among the retrieval cues.

Almost without exception, interpretations of so-called long-term memory factors on immediate memory call upon some form of interaction between the more recent representations associated with the current trial and the representations that participants bring to the laboratory. This is true of the various versions of the reconstruction hypothesis, of the feature model, and of others. In many cases, the assumption is that there is reconstruction or retrieval of a recall candidate from long-term memory based on a constellation of cues that reflect the characteristics of the immediate memory trial. The data reported here provide a first assessment of the nature of the search set. Our results are entirely compatible with the assumption that in the absence of some more restric- tive cue, the set of competitors available for retrieval is constrained only by the characteristics of the degraded cue called upon to support retrieval.

\section{REFERENCES}

Brooks, J. O., \& WatKIns, M. J. (1990). Further evidence of the intricacy of memory span. Journal of Experimental Psychology: Learning, Memory, \& Cognition, 16, 1134-1141.

Brown, G. D. A., \& Hulme, C. (1995). Modeling item length effects in memory span: No rehearsal needed? Journal of Memory \& Language, 34, 594-621.

Crowder, R. G. (1979). Similarity and order in memory. In G. H. Bower (Ed.), The psychology of learning and motivation: Advances in research and theory (Vol. 13, pp. 319-353). New York: Academic Press.

Estes, W. K. (1991). On types of item coding and sources of recall in short-term memory. In W. E. Hockley \& S. Lewandowsky (Eds.), Relating theory and data: Essays on human memory in honor of Bennet B. Murdock (pp. 175-194). Hillsdale, NJ: Erlbaum.

Hulme, C., Maughan, S., \& Brown, G. D. A. (1991). Memory for familiar and unfamiliar words: Evidence for a long-term memory contribution to short-term memory span. Journal of Memory \& Language, 30, 685-701.

Hulme, C., Stuart, G., Brown, G. D. A., \& Morin, C. (2003). Highand low-frequency words are recalled equally well in alternating lists: Evidence for associative effects in serial recall. Journal of Memory \& Language, 49, 500-518.

Loftus, G. R., \& MAsson, M. E. J. (1994). Using confidence intervals in within-subject designs. Psychonomic Bulletin \& Review, 1, 476-490.

Multhaup, K. S., Balota, D. A., \& Cowan, N. (1996). Implications of aging, lexicality, and item length for the mechanisms underlying memory span. Psychonomic Bulletin \& Review, 3, 112-120.

Murdock, B. B., JR. (1976). Item and order information in short-term serial memory. Journal of Experimental Psychology: General, $\mathbf{1 0 5}_{2}$ 191-216.

NAIRNE, J. S. (1990). A feature model of immediate memory. Memory \& Cognition, 18, 251-269.

NEATH, I. (1994, May). Temporal encoding and the auditory superiority hypothesis. Paper presented at the 66th meeting of the Midwestern Psychological Association, Chicago.

Neath, I., \& Crowder, R. G. (1990). Schedules of presentation and temporal distinctiveness in human memory. Journal of Experimental Psychology: Learning, Memory, \& Cognition, 16, 316-327.

NEATH, I., \& NAIRNE, J. S. (1995). Word-length effects in immediate memory: Overwriting trace decay theory. Psychonomic Bulletin \& Review, 2, 429-441.

PoIRIER, M., \& SAINT-Aubin, J. (1995). Memory for related and unrelated words: Further evidence on the influence of semantic factors in immediate serial recall. Quarterly Journal of Experimental Psychology, 48A, 384-404.

RoODEnRys, S., \& Quinlan, P. T. (2000). The effects of stimulus set size and word frequency on verbal serial recall. Memory, 8, 71-78.

Saint-Aubin, J., \& PoIrIer, M. (1999). Semantic similarity and immediate serial recall: Is there a detrimental effect on order information? Quarterly Journal of Experimental Psychology, 52A, 367-394.

SAint-Aubin, J., \& Poirier, M. (2000). Immediate serial recall of words and nonwords: Tests of the retrieval-based hypothesis. Psychonomic Bulletin \& Review, 7, 332-340.

SAINT-Aubin, J., \& PoIrIER, M. (in press). Word frequency effects in immediate serial recall: Item familiarity and item co-occurrence have the same effect. Memory.

SCHWEICKERT, R. (1993). A multinominal processing tree model for degradation and redintegration in immediate recall. Memory \& Cognition, 21, 168-175.

STUART, G., \& HULME, C. (2000). The effects of word co-occurrence on short-term memory: Associative links in long-term memory affect short-term memory performance. Journal of Experimental Psychology: Learning, Memory, \& Cognition, 26, 796-802.

Tehan, G., \& HumPhreYs, M. S. (1995). Transient phonemic codes and immunity to proactive interference. Memory \& Cognition, 23, 181-191. 
Tolan, G. A., \& Tehan, G. (1999). Determinants of short-term forgetting: Decay, retroactive interference, or proactive interference? International Journal of Psychology, 34, 285-292.

\section{NOTE}

1. It is worth noting that contrary to the present study, Tehan and Humphreys's (1995) standard procedure is not an immediate serial recall task. Instead, a typical trial involves the presentation of two blocks of four items, with a short pause between them. Both blocks are assembled in order to maximize interference among them by placing, at the same serial position within both blocks, a member of the same taxonomic or phonological category. For instance, in Tolan and Tehan's (1999) first experiment, a high-dominant member of a category was placed in the first (interference) block and a relatively weak member of the same category was placed in the second block. Presentation was followed by a retention interval, during which a secondary task was performed to degrade phonological representations. At recall, participants were asked to recall either the first or the second block. Proactive interference was evidenced when the second block was cued for recall and a word from the first block was recalled. In the present study, the lack of proactive interference effects even under suppression was likely due to the construction of our lists, which - as it is usually the case in the field-was not aimed at maximizing proactive interference.

(Manuscript received January 15, 2004; revision accepted for publication April 16, 2004.) 